\title{
Blattella germánica (Blattodea: Blattellidae) como potencial vector mecánico de infecciones asociadas a la atención en salud (IAAS) en un centro hospitalario de Villavicencio (Meta-Colombia)
}

Blattella germanica (Blattodea: Blattellidae) as potential mechanical vector of health-care associated infections in a hospital in Villavicencio (Meta-Colombia).

Gloria Isabel Jaramillo $\mathrm{PhD}^{1 *}$, Norma Cristina Pavas MSc ${ }^{1}$, Juan Camilo Cárdenas ${ }^{1}$, Paola Gutiérrez ${ }^{1}$, William Andrés Oliveros $^{1}$, María Angélica Pinilla ${ }^{1}$

\section{Resumen}

Objetivo. Identificar las especies de cucarachas y bacterias asociadas a su exoesqueleto en un centro hospitalario de la ciudad de Villavicencio (Meta, Colombia). Método. Se realizaron capturas manuales de cucarachas en cocina, urgencias, UCI intermedia, consulta externa y neonatos. Los individuos colectados fueron sometidos a un aislamiento primario en caldo BHI, para luego pasar a medios sólidos (sangre y MacConkey). Se realizó identificación y antibiograma por método automatizado. Resultados. Se colectaron 24 ninfas y adultos de Blattella germánica. Se aisló e identificó Klebsiella pneumoniae con sensibilidad intermedia a meropenem (CMI 4) y resistencia a cefalosporinas (cefepima y cefuroxima) (>16), Proteus vulgaris con resistencia a cefalosporinas (ceftriaxona, cefuroxima) (CMI $>16)$, Enterobacter cloacae con resistencia a cefalosporinas (cefoxitina, cefuroxima, ceftriaxona) (CMI > 16), Enterococcus faecium, Enterococcus rafinosus, Staphylococcus xylosus y Enterococcus faecalis. El 80\% de las bacterias aisladas presentaron algún grado de resistencia a antibióticos. Conclusiones. Estos insectos podrían jugar un papel importante en la transmisión de las Infecciones asociadas a la atención en salud (IAAS). Debido a su presencia en hospitales y el reporte de IAAS y resistencia bacteriana en varios centros asistenciales en el Departamento del Meta, se hace necesario establecer la relación de estos insectos con estos eventos.

Palabras clave: Antibióticos, cucarachas, infección nosocomial, microorganismos, susceptibilidad, resistencia

\section{Abstract}

Objective. Identify the species of cockroaches and bacteria associated with their exoskeleton in a hospital in Villavicencio (Meta, Colombia). Methods. The cockroaches were captured manually in kitchen, emergency room, intermediate intensive care unit, outpatient service and newborn nursery. The collected individuals were subjected to a primary isolation in $\mathrm{BHI}$ broth, then move on to solid media (blood and MacConkey). Identification and sensitivity testing was performed by automated method. Results. 24 nymphs and adults of Blattella germanica were collected. It was isolated and identified Klebsiellapneumoniae with intermediate susceptibility to meropenem (MIC 4) and resistance to cephalosporins (cefepime and cefuroxime) (> 16), Proteus vulgaris with cephalosporin resistance (Ceftriaxone, Cefuroxime) (MIC >

${ }^{1}$ Facultad de Medicina, Universidad Cooperativa de Colombia, Villavicencio, Colombia 
16), Enterobacter cloacae resistant to cephalosporins (cefoxitin, cefuroxime, ceftriaxone) (MIC > 16), Enterococcus faecium, Enterococcus rafinosus, Staphylococcus xylosus and Enterococcus faecalis. 80\% of the isolated bacteria showed some degree of resistance to antibiotics. Results. These insects could play an important role in the transmission of health care associated infections (HCAI). Due to the presence of cockroaches in hospitals and the report of HCAI and bacterial resistance in several health centers in the Department of Meta, it is necessary to establish the relationship of these insects with these events.

Key words: Antibiotics, cockroaches, nosocomial infection, microorganisms, susceptibility, resistance

\section{Introducción}

Las Infecciones asociadas a la atención en salud (IAAS), conocidas también como infecciones hospitalarias o nosocomiales, son procesos localizados o sistémicos adquiridos durante una estancia en cualquier centro de atención en salud (1), así como la reacción adversa debida a la presencia de un microorganismo o sus toxinas, que se presenta durante o hasta 72 horas después del egreso de la misma (2-3).

Estas infecciones son consideradas un problema de salud pública ya que son de difícil manejo y muchas veces asociadas a microorganismos resistentes a los antimicrobianos (4). También son considerados de gran interés epidemiológico, debido no solo a la morbilidad y mortalidad asociada sino también a las pérdidas económicas que conllevan (5).

En Colombia los organismos más prevalentes asociados a IAAS son E. Coli $(52,7 \%)$, Klebsiella pneumoniae (12,8\%), Staphylococcus aureus $(9,1 \%)$, Pseudomonas aeruginosa (6,9\%), Enterococcus faecalis (5,4\%), Proteus mirabilis (4,7\%), Staphylococcus epidermidis (3,7\%), E. Cloacae $(3,1 \%)$, Staphylococcus hominis $(1,1 \%)$ y Acinetobacter sp $(0,6 \%)(6,7)$.

El departamento del Meta en el año 2015 presentó una resistencia importante en enterobacterias; la resistencia de E. Coli, Klebsiella pneumoniae y E. Cloacae, lo posicionó como uno de los departamentos con más altos porcentajes de resistencia a cefalosporinas de tercera generación y a carbapenémicos. En cuanto a microorganismos no fermentadores,
Pseudomonas aeruginosa (35\%) y Acinetobacter baumannii (54\%) reportaron los mayores porcentajes de resistencia a carbapenémicos, superando los niveles nacionales. Para los microorganismos Gram positivos se observó la presencia de Staphylococcus aureus meticilino resistente, con un porcentaje de resistencia para el departamento del Meta del $36 \%$ superando el nivel nacional y posicionándose junto con Barranquilla y Santander en los primeros lugares de resistencia (8).

La presencia de insectos en áreas intrahospitalarias representa un potencial riesgo para la salud de los pacientes, debido a que han sido implicados en la transmisión mecánica de microorganismos patógenos al hombre. Estos artrópodos pueden poner en riesgo la higiene de estos establecimientos gracias a la gran cantidad de hábitats y hábitos alimenticios que presentan algunas especies (9). Estas condiciones los ponen en contacto con agentes patógenos como virus, bacterias, protozoos y helmintos que pueden ser transmitidos mecánicamente por los insectos y causar infecciones asociadas a la atención en salud (IAAS) (10).

Las cucarachas, insectos pertenecientes al orden Blattodea, son considerados como vectores mecánicos de microorganismos como E. Coli, Pseudomonas aeruginosa, Salmonella, Listeria monocytogenes y conidias de hongos micotoxigénicos entre otros (3). En términos generales, se las ha implicado en la transmisión de 150 especies de bacterias, 60 especies de levaduras, 90 especies de protozoos y 45 especies de parásitos (11). En el mundo existen alrededor de 4000 especies de cucarachas, sin embargo, solo 
el $1 \%$ se encuentra relacionado con los Humanos. A pesar del relativo bajo porcentaje de potenciales especies vectoras, es considerado uno de los agentes más importantes que pueden ser infectados con bacterias patogénicas causantes de lepra, peste bubónica, disentería, infecciones urinarias, abscesos (11).

Considerando la importancia de las cucarachas en la transmisión mecánica de microorganismos patógenos en hospitales (12), y de la presencia de IAAS y resistencia bacteriana en varios centros asistenciales del Departamento, se hace necesario realizar este tipo de estudios que permitan establecer la relación de estos insectos con IAAS, para poder tomar medidas de control necesarias y eficientes que bajen los niveles de infestación de forma segura y disminuir el riesgo a los pacientes. Este estudio piloto tuvo como objetivo identificar las especies de cucarachas y las bacterias asociadas a su exoesqueleto en un centro hospitalario de la ciudad de Villavicencio (Meta, Colombia).

\section{Materiales y métodos}

Se realizó un estudio piloto de tipo observacional, descriptivo, transversal, a partir de muestras bacterianas aisladas de cucarachas colectadas en diferentes áreas de atención de un centro hospitalario de la ciudad de Villavicencio (Meta). Las áreas para el estudio fueron seleccionadas de acuerdo a los permisos proporcionados por la Administración de la clínica: cocina, urgencias, UCI intermedia, consulta externa y sala de partos. La búsqueda y captura de insectos se realizó en zonas como paredes, tomacorrientes, grietas, equipos electrónicos y estantes. Se realizó captura manual teniendo en cuenta la bioseguridad utilizando guantes, pinzas y pinceles estériles.

Una vez capturado el individuo era individualizado en un tubo eppendorf estéril y rotulado para su identificación. Los insectos recolectados fueron sometidos inmediatamente a una temperatura de $4^{\circ} \mathrm{C}$ por 20 minutos, para inmovilizarlos y posteriormente fueron puestos en caldo BHI (medio de enriquecimiento), por una hora. Luego las cucarachas fueron retiradas del medio enriquecido, el cual siguió en incubación por 24 horas. Posteriormente se pasaron $100 \mu \mathrm{l}$ de este caldo a medios solidos (agar sangre y MacConkey) los cuales se incubaron de 24 a 48 horas para la obtención de aislamientos bacterianos.

Las cepas bacterianas fueron llevadas en medios de transporte (Aimes y Cary Blair) al Laboratorio Clínico de la Corporación Clínica Universidad Cooperativa de Colombia (CCUCC) en donde se realizó la identificación y antibiograma de los microorganismos por método automatizado (Beckton Dickinson), incorporando los datos obtenidos en un ordenador con un software integrado con la BD EpiCenter ${ }^{\mathrm{TM}}$, que proporciona un alto índice de exactitud a la identificación de los mismos. Se realizó la determinación del estado de susceptibilidad o resistencia de las bacterias aisladas a partir de los antibióticos Amikacina, Amoxicilina-clavulonato, Ampicilina, Cefepima, Cefoxitina, Ceftriaxona, Cefuroxima, Ciprofloxacina, Ertapenem, Gentamicina, Imipenem, Levofloxacino, Meropenem, Piperacilina-Tazobactam, Tigeciclina, Trimetropin-Sulfametoxazol.

Análisis estadístico: Se realizó un análisis descriptivo de los datos teniendo en cuenta el tipo de variable y su naturaleza, utilizando Excel para la tabulación de los mismos.

Consideraciones éticas: Para la realización del estudio se contó con los avales institucionales y de los comités de ética de la Universidad Cooperativa de Colombia, sede Villavicencio y del centro hospitalario. Para el manejo de los microorganismos se siguieron las disposiciones de la resolución 008430 de 1993 en su Título IV, Capítulo I. Se contó con laboratorios y equipos necesarios para la realización de pruebas microbiológicas bajo las condiciones de bioseguridad necesarias. 


\section{Resultados}

Se colectaron un total de 24 individuos en las diferentes áreas de la clínica. Todas las cucarachas colectadas pertenecían a la especie Blattella germánica. Se capturaron tanto ninfas (de diferentes estadíos) como adultos. En la sala de partos se capturaron once ninfas, en urgencias se colectaron tres ninfas y una hembra adulta y en la cocina se capturaron diez ninfas y una hembra adulta con la ooteca aun pegada a su abdomen. Los individuos fueron encontrados en gabinetes, detrás de muebles y equipos, cerca de lugares de almacenamiento de desechos. En la cocina se las encontró próximas a residuos de alimentos, en cartones de huevos y en grietas en las paredes. En consulta externa y UCI intermedia no se encontraron individuos, sin embargo, en esta última, se encontraron excretas de estos insectos en una de las unidades. Debido al tamaño de muestra y la cantidad de medios disponibles, se realizaron grupos de individuos dependiendo del sitio de captura para su análisis microbiológico.

Del exoesqueleto de las cucarachas capturadas en la cocina se aislaron e identificaron las siguientes bacterias: Klebsiella pneumoniae, Proteus vulgaris,
Enterococcus faecium y E. Cloacae. En el área de urgencias se aisló Klebsiella pneumoniae, Enterococcus rafinosus y Staphylococcus xylosus; y en la sala de partos E. Cloacae y Enterococcus faecalis. De igual manera se realizó el aislamiento e identificación de Bacillus cereus de una muestra de hormigas (Tapinoma melanocephalum) colectadas en consulta externa.

El $80 \%$ de las bacterias aisladas presentaron algún grado de resistencia a antibióticos. De la batería de 16 antibióticos probados en el estudio, Klebsiella pneumoniae ha desarrollado algún grado de resistencia a siete de ellos, seguido de E. Cloacae con resistencia a seis antibióticos. Staphylococcus xylosus, el cual solo fue aislado en urgencias, presentó resistencia únicamente a la ampicilina, Tabla 1.

Dentro de los resultados más representativos, se observó que Klebsiella pneumoniae presentó una sensibilidad intermedia a meropenem (CMI 4) y resistencia a cefalosporinas (cefepima y cefuroxima) (>16). E. Cloacae presentó resistencia a cefalosporinas (cefoxitina, cefuroxima, ceftriaxona) (CMI > 16). Y Proteus vulgaris presentó resistencia a cefalosporinas (ceftriaxona, cefuroxima) (CMI $>16)$, Tabla 1.

Tabla 1. Estado de la resistencia a antibióticos de las bacterias aisladas del exoesqueleto de Blattella germánica en diferentes áreas de un centro hospitalario en la ciudad de Villavicencio (Meta, Colombia).

\begin{tabular}{|c|c|c|c|c|}
\hline Sitio de colecta & Bacteria identificada & Antibiótico & Estado* & $\mathrm{CMI}^{* *}$ \\
\hline \multirow{9}{*}{ Cocina } & \multirow{5}{*}{ Klebsiella pneumoniae } & Cefepima & $\mathrm{r}$ & $>16$ \\
\hline & & Meropenem & $\mathrm{i}$ & 4 \\
\hline & & Ampicilina & $\mathrm{r}$ & $>16$ \\
\hline & & Cefuroxima & $\mathrm{r}$ & $>16$ \\
\hline & & Tigeciclina & $\mathrm{i}$ & 4 \\
\hline & \multirow{4}{*}{ Proteus vulgaris } & Ampicilina & r & $>16$ \\
\hline & & Ceftriaxona & r & 32 \\
\hline & & Cefuroxima & $\mathrm{r}$ & $>16$ \\
\hline & & Tigeciclina & $\mathrm{r}$ & \\
\hline
\end{tabular}




\begin{tabular}{|c|c|c|c|c|}
\hline \multirow{6}{*}{ Cocina } & \multirow{2}{*}{ Enterococcus faecium } & Ampicilina & $\mathrm{r}$ & $>8$ \\
\hline & & Linezolid & $\mathrm{i}$ & 4 \\
\hline & \multirow{4}{*}{ E. Cloacae } & Amoxicilina-clavulonato & $\mathrm{r}$ & $>16 / 8$ \\
\hline & & Ampicilina & $\mathrm{r}$ & $>16$ \\
\hline & & Cefoxitina & $\mathrm{r}$ & $>16$ \\
\hline & & Cefuroxima & $\mathrm{r}$ & $>16$ \\
\hline \multirow{5}{*}{ Urgencias } & \multirow{4}{*}{ Klebsiella pneumoniae } & Amoxicilina-clavulonato & $\mathrm{r}$ & $>16 / 8$ \\
\hline & & Ampicilina & $\mathrm{r}$ & $>16$ \\
\hline & & Cefoxitina & $\mathrm{r}$ & $>16$ \\
\hline & & Cefuroxima & $\mathrm{r}$ & $>16$ \\
\hline & Staphylococcus xylosus & Ampicilina & $\mathrm{r}$ & \\
\hline \multirow{6}{*}{ Sala parto } & \multirow{6}{*}{ E. Cloacae } & Amikacina & $\mathrm{i}$ & 32 \\
\hline & & Amoxicilina-clavulonato & $\mathrm{r}$ & $>16 / 8$ \\
\hline & & Ampicilina & $\mathrm{r}$ & $>16$ \\
\hline & & Cefoxitina & $\mathrm{r}$ & $>16$ \\
\hline & & Ceftriaxona & $\mathrm{r}$ & 32 \\
\hline & & Cefuroxima & $\mathrm{r}$ & $>16$ \\
\hline
\end{tabular}

${ }^{*} \mathrm{r}=$ resistente; $\mathrm{i}=$ intermedio

${ }^{* *} \mathrm{CMI}=$ Concentración mínima inhibitoria

\section{Discusión}

En este estudio piloto realizado en un centro hospitalario de segundo nivel de la ciudad de Villavicencio, se investigó la flora microbiana aislada del cuerpo de cucarachas colectadas en diferentes áreas del mismo. Estas observaciones han sido descritas por otros autores en diversas partes del mundo, la mayor producción literaria sobre el tema se concentra en el continente Africano (1316) y Oriente Medio $(3,11,12,17,18)$.

Los resultados mostraron que las cucarachas son capaces de alojar y transportar una gran cantidad de bacterias patógenas, en su gran mayoría con algún grado de resistencia a uno o más antibióticos, Tabla 1. Tilahun y colaboradores encontra- ron quince especies de bacterias en una unidad neonatal en Addis Ababa, las cuales presentaron resistencia múltiple a los doce antibióticos probados (14). En Ghana se realizó un estudio en un hospital de tercer nivel, en donde se hallaron ocho tipos de bacterias, las cuales presentaron diferentes niveles de resistencia a antimicrobianos (13). Estos hallazgos indican que las cucarachas podrían ser un vector mecánico potencial de bacterias resistentes asociadas con IAAS $(14,19)$, con lo cual se deben extremar medidas para su control efectivo dentro de las instituciones prestadoras de servicios de salud.

Durante el muestreo realizado solo se encontraron individuos de Blattella germánica en todas las áreas revisadas. Esta especie ya ha sido reportada en la 
ciudad de Cali (Colombia) con altos grados de infestación en áreas intrahospitalarias, en donde se la encontró en salas de pediatría en camas, marcos de puertas y ventanas, estantes e instalaciones eléctricas (20). A pesar de estos resultados, es muy probable que también se encuentren otras especies de cucarachas co-habitando las instalaciones de la clínica, ya que las condiciones de este tipo de edificaciones en conjunto con el clima tropical de la ciudad proporcionan un hábitat propicio para estos insectos. Especies como Periplaneta americana y $P$. australiaseae, son muy comunes en nuestro medio, altamente sinantrópicas y cosmopolitas; además son capaces de adaptarse a diversos ambientes tanto en el intra como en el peridomicilio $(21,22)$ y han sido involucradas por otros autores como posibles vectores mecánicos de microorganismos patógenos relacionados con IAAS $(3,12,23,24)$.

La concentración mínima Inhibitoria (CMI) que corresponde a la base de la medida de la sensibilidad bacteriana a un antibiótico constituye la escala en que se puede medir la actividad del antibiótico probado, categorizando la bacteria en sensible (s) o resistente (r) e intermedia (i) (25). Las bacterias aisladas en el presente estudio, se encuentran enumeradas con las IAAS en Colombia y se encuentran relacionadas en el protocolo de resistencia bacteriana a los antimicrobianos en el ámbito hospitalario del Instituto Nacional de Salud (INS) (26 - 29). Klebsiella pneumoniae, E. Cloacae y Enterococcus faecium tienen la capacidad de generar resistencia a antimicrobianos y diseminar plásmidos de resistencia a otros géneros, que complican la situación en la institución y deben estar sujetos a seguimiento y control institucional. El fenotipo de resistencia encontrado en $K$. pneumoniae (resistencia a cefalosporinas y carbapenémicos intermedio) establece un perfil con criterio para ser confirmado y vigilado dado que se relaciona con la presencia de carbapenemasas que constituyen una emergencia hospitalaria y una grave amenaza para la salud de los pacientes y la salud pública.
Las cucarachas de la especie Blattella germanica, son capaces de transportar en su cuerpo diversas especies de bacterias patógenas, algunas de ellas con altos niveles de resistencia a diversos antimicrobianos; estos hallazgos preliminares podrían indicar que estos insectos están jugando un papel importante en las infecciones asociadas a la atención en salud en la ciudad de Villavicencio. Por lo tanto, las instituciones prestadoras de servicios de salud, deben realizar un manejo integrado tanto de los insectos plaga como de las bacterias, para disminuir el riesgo de transmisión de estas infecciones.

\section{Agradecimientos}

Los investigadores agradecen a la Corporación Clínica Universidad Cooperativa de Colombia, sede Villavicencio (Meta), por facilitar la realización de los muestreos, identificación y antibiograma de los microorganismos.

\section{Referencias}

1. Shukla P, Garg R, Dahiya A. Role of technology to combat nosocomial infections. Apollo Medicine. 2015; 338: 1-3.

2. Secretaria Distrital de Salud de Bogotá. Sistema de Vigilancia epidemiológica de infecciones intrahospitalarias.

3. Kassiri H, Kassiri A, Kazemi S. Investigaction on American cockroaches medically important bacteria in Khorramshahr hospital, Iran. Asian Pacific Journal of tropical Disease. 2014; 4(3): 201-203.

4. Rivera SM. Informe final infecciones asociadas a dispositivos, Colombia 2013. Informe evento. Bogotá: Instituto Nacional de Salud; 2013. Report No.: FOR-R02.4000-001.

5. Oliveira P, Souza S, Campos G, Da Silva D, Sousa D, Araujo $S$, et al. Isolation, pathogenicity and disinfection of Staphylococcus aureus carried by insects in two public hospitals in Vitoria da Conquista, Bahia, Brazil. Brazilian Journal Infection Diseases. 2014; 18(2): 129-136.

6. RENOVA. Informe microbiolópgico 2010-2012. 2012.

7. Instituto Nacional de Salud, Dirección de Redes en Salud Pública. Vigilancia de Resistencia Bacteriana a través de las bases de datos whonet. Ańo 2013. 
8. Instituto Nacional de Salud. Vigilancia de Resistencia bacteriana Whonet - I semestre 2015. 2015 Diciembre.

9. Olaya LA, Chacón de Ulloa P, Payán A. Hormigas (Hymenoptera: Formicidae) en centros hospitalarios del Valle del Cauca como vectores patógenos nosocomiales. Revista Colombiana de Entomología. 2005; 31(2): 183-187.

10. Trasmonte A, García Y, Humbria L, García de Humbría G, Cazorla D. Aislamiento de enterobacterias en la mosca común (Musca domestica) en Coro, estado de Falcón, Venezuela. Boletín de Malariología y Salud Ambiental. 2009; XLIX(2): 275-281.

11. Jalil N, Amir K, Hasan MK, Mahdi M, Monireh M, Atefeh B. Cockroaches bacterial infections in wards of hospitals, Hamedan city, west of Iran. Asian Pacific Journal of Tropical Disease. 2012; 381-384.

12. Ghasemi_Dehkordi P, Doosti A, Doosti E, Noshadi E, Arshi A. Antimicrobial susceptibility patterns of Escherichia coli isolates from cockroaches in southwestern Iran. Bulgarian Journal of Veterinary Medicine. 2015; 1-9.

13. Tetteh-Quarcoo P, Donkor E, Attah S, Duedu K, Afutu E, Boamah I, et al. Microbial carriage of cockroaches at a tertiary care hospital in Ghana. Environmental Health Insights. 2013; 7: 59-66.

14. Tilahun B, Worku B, Tachbele E, Terefe S, Kloos H, Legesse W. High load of multu-drug resistant nosocomial neonatal pathogens carried by cockroach in a neonatal intensive care unit at Tikur Anbessa specialized hospital, Addis Ababa, Ethiopia. Antimicrobial Resistance and Infection Control. 2012; 1(12): 1-7.

15. Menasria T, Tine S, Hamza S, Mahcene D, Moussa F, Benammar L, et al. A survey of the possible role of german cockroaches as a source for bacterial pathogens. Journal of Advanced Sciences \& Applied Engineering. 2014; 1(1): 67-70.

16. Adeleke MA, Akatah HA, Hassan AO, Sam-Wobo SO, Famodimu TM, Olatunde GO, et al. Implication of cockroaches as vectors of gastrointestinal parasites in parts os osogobo, southwestern Nigeria. Munis Entomology \& Zoology. 2012; 7(2): 1106-1110.

17. Handool H, Al.Aridhi H. Antibiotic resistant bacteria associated with the cockroach Periplaneta americana in Al-Diwaniya city /Iraq. International Journal of Advnaced Research. 2014; 2(2): 709-7014.

18. Salehzadeh A, Tavacol P, Mahjub H. Bacterial, fungal and parasitic contamination of cockaroaches in public hospitals of Hamadan, Iran. Journal of Vector Borne Disease. 2007; 44: 105-110.

19. Menasria T, Moussa F, El-Hamza S, Tine S, Megri R, Chenchouni H. Bacterial load of german cockroach (Blattella germanica) found in hospital environment. Pathogens and Global Health. 2014; 108(3): 141-147.

20. Piazuelo MM, Jaramillo I, Gonzalez R. Resistencia a deltametrina de cepas de Blattella germanica (Dyctioptera: Blattellidae) en la ciudad de Cali, Colombia. Revista Cubana de Medicina Tropical. 2009; 61(3): 213-219.

21. Xue FU, Lefu YE, Feng GE. Habitat influences on diversity of bacteria found on german cockroach in Beijing. Journal of Environmentla Sciences. 2009; 21: 249-254.
22. Jaramillo I, Cardenas H, Gonzalez R, Rosero C. Genetic variability of five Periplaneta americana 1. (Dyctiopteria: Blattellidae) populations in southwestern Colombia using the AFLP molecular marker technique. Neotropical Entomology. 2010; 39(3): 371-378.

23. Akinjogunla OJ, Odeyemi AT, Udoinyang EP. Cockroaches (Periplaneta americana and blattella germanica): reservoirs of multi drug resistant (MDR) bacteria in Uyo, Akwa Ibom State. Scientific Journal of Biological Sciences. 2012; 1(2): 19-30.

24. Kassiri H, Kazemi S. Cockroaches (Periplaneta americana (L.), Dyctioptera; Blattellidae) as carriers of bacterial pathogens, Khorramshahr County, Iran. Jundishapur Journal of Microbiology. 2012; 5(1): 320-322.

25. Cercenado E, Saavedra J. El antibiograma. Interpretación del antibiograma, conceptos generales. Annales de Pediatria Continuada. 2009; 7(4): 214-217.

26. Instituto Nacional de Salud. Resistencia bacteriana a los antimicrobianos en el ámbito hospitalario. protocolo de Vigilancia en Salud Pública. Bogotá: Instituto Nacional de Salud; 2014. Report No.: PRO-R02-043.

27. Galvez, Z. Y. A. and V. E. M. Burbano (2015). "Bacillus: género bacteriano que demuestra ser un importante solubilizador de fosfato.” NOVA Publicación en Ciencias Biomédicas.

28. Ramírez, L. C. C., et al. (2014). "Determinación de la presencia de bacterias patógenas para el humano en aguas de riego en la cuenca alta de la sabana de Bogotá; DC Colombia." Nova $12(22)$.

29. Campuzano, S., et al. (2015). "Determinación de la calidad microbiológica y sanitaria de alimentos preparados vendidos en la vía pública de la ciudad de Bogotá DC.” Nova. 